\title{
Abordaje quirúrgico de la fístula traqueoesofágica iatrogénica. Presentación de caso y revisión de la literatura
}

\author{
Surgical approach for iatrogenic tracheoesophageal fistula. Case report and literature \\ review
}

\author{
Jesús F. Nagore-Ancona*, Arturo Acosta-Martínez, Josúe A. González-Luna, Jesús A. Martín-Pérez, \\ Ana L. Sánchez-Baltazar y Luisana Perna-Lozada
}

Servicio de Cirugía General, Hospital Regional General Ignacio Zaragoza, Instituto de Seguridad y Servicios Sociales de los Trabajadores del Estado, Ciudad de México, México

\section{Resumen}

Antecedentes: La fístula traqueoesofágica es una complicación en pacientes con ventilación mecánica prolongada. La contaminación pulmonar permanente puede ser grave, con evolución fatal. Objetivo: Describir un caso de fístula traqueoesofágica posintubación y hacer una breve revisión de la literatura. Caso clínico: Mujer de 45 años que sufre un evento cerebrovascular isquémico que condiciona deterioro neurológico progresivo, ameritando ventilación mecánica prolongada. Durante la gastrostomía endoscópica se evidencia una fístula. Resultados: Intervenida quirúrgicamente para reparación esofágica y resección traqueal mediante cervicoesternotomía, lamentablemente fallece en el posoperatorio inmediato. Conclusiones: Esta patología es compleja. Deben implementarse protocolos enfocados en su prevención en pacientes en quienes se espera una ventilación mecánica prolongada.

Palabras clave: Fístula traqueoesofágica. Enfermedades de la tráquea. Esternotomía media. Ventilación mecánica.

\begin{abstract}
Background: Post-intubation tracheoesophageal fistula is a severe complication in long-term mechanical ventilation patients with possible fatal consequences. Objective: To describe a case of post-intubation tracheoesophageal fistula and its surgical management. In addition, a brief literature review was effectuated. Case report: 45-year-old female, suffers ischemic stroke with progressive neurological damage that requires long-term mechanical ventilation. During endoscopic gastrostomy tracheoesophageal fistula is shown. Results: Undergoes surgery for esophageal repair and tracheal resection, through cervicosternotomy. Unfortunately died in the immediate postoperative period. Conclusions: This complex pathology requires structured protocols for its prevention in patients in whom long-term mechanical ventilation is expected.
\end{abstract}

Key words: Tracheoesophageal fistula. Tracheal diseases. Median sternotomy. Mechanical ventilation.

Cir Cir. 2021;89(6):811-817 Contents available at PubMed www.cirugiaycirujanos.com 0009-7411/@ 2020 Academia Mexicana de Cirugía. Publicado por Permanyer. Este es un artículo open access bajo la licencia CC BY-NC-ND (http://creativecommons.org/licenses/by-nc-nd/4.0/).
} 


\section{Introducción}

La fístula traqueoesofágica en adultos ocurre como resultado de traumatismo, malignidad, necrosis traqueal inducida por sobreinsuflación del globo de la cánula endotraqueal en casos de ventilación mecánica prolongada, intubación endotraqueal traumática, ingestión de cuerpo extraño, mediastinitis o presencia prolongada de sonda nasogástrica rígida, o como complicación quirúrgica de una resección traqueal o una cirugía esofágica o mediastinal ${ }^{1}$.

Se estima que su frecuencia oscila entre el $0.3 \%$ y el $4 \%$ de todos los pacientes intubados y sometidos a ventilación mecánica ${ }^{2,3}$. Su fisiopatología es variable y ligada al mecanismo de lesión. En los casos con ventilación mecánica prolongada, la fístula traqueoesofágica es inducida por isquemia de la pared posterior de la tráquea causada por la sobreinsuflación del balón endotraqueal, que se encuentra en contacto con una sonda nasogástrica utilizada para alimentar o para aspirar el contenido gástrico; la necrosis de la pared con la fístula secundaria se puede presentar cuando esta condición se prolonga más de 2 horas. Los factores de riesgo son la presión elevada sobre la vía aérea, la movilidad excesiva de la cánula endotraqueal, el tiempo prolongado de intubación, el tratamiento con esteroides, la diabetes dependiente de insulina, el pobre estado nutricional, la hipoxia crónica en enfermedades cardiopulmonares, los episodios prolongados de hipotensión, la anemia crónica, la sepsis y el reflujo gastroesofágico ${ }^{2,4}$.

La lesión iatrogénica puede ser resultado de una intubación de emergencia, con lesiones distintas a las que se presentan en la lesión traumática asociada a ventilación mecánica. Se caracteriza por una laceración longitudinal de la pared posterior traqueal. El proceso inflamatorio es progresivo, inducido por el balón endotraqueal, por lo que nunca se presentan fugas hacia el mediastino, tal como se observa en la fístula traumática. La inflamación perilesional une las paredes traqueal y esofágica, por lo que nunca ocurre mediastinitis, ${ }^{5,6}$.

La fístula traqueoesofágica puede desarrollarse tras 12 a 200 días de ventilación mecánica, con una media de 40 días, aunque el tiempo en que puede volverse sintomática es variable de acuerdo con la etiología, la localización y el tamaño de la lesión'1. Esto también condiciona la gravedad de la clínica, la urgencia, la elección del tratamiento y el pronóstico final. La resolución pronta es importante, ya que la contaminación pulmonar permanente por residuos alimentarios y secreciones digestivas puede ser muy grave, con una posible evolución fatal ${ }^{4}$.

El objetivo del presente trabajo es describir un caso clínico de fístula traqueoesofágica posintubación y el manejo quirúrgico realizado.

\section{Caso clínico}

Mujer de 45 años, sin antecedentes de importancia, que ingresa al servicio de urgencias por presentar alteración de la marcha de forma súbita. En la exploración física está alerta, consciente, orientada, sin datos de focalización, hemodinámicamente estable, con bradicardia sinusal, pero sin datos de bajo gasto, con mecánica ventilatoria conservada y abdomen sin datos patológicos. Al ingreso se le realiza una tomografía computarizada de cráneo que evidencia un evento vascular cerebral de tipo isquémico, con edema que desplaza la línea media. Valorada por el servicio de neurocirugía, se concluye que no es candidata a manejo quirúrgico. Dos días después presenta un nuevo deterioro neurológico agudo, con una puntuación en la Escala de Coma de Glasgow de 6 puntos (01, V1, M4): localiza el dolor, pupilas midriáticas, sin respuesta a estímulos luminosos. Se procede a manejo avanzado de la vía aérea con apoyo mecánico ventilatorio, cursando con evolución tórpida hacia un deterioro sin posibilidad de progresión para el destete de la ventilación mecánica, desarroIlando neumonía asociada a ventilación mecánica y choque séptico secundario. Cuarenta y cinco días después de la intubación se le coloca una sonda de gastrostomía por endoscopia, que identifica una fístula traqueoesofágica compleja (Fig. 1). La tomografía computarizada cervical reporta un aumento del diámetro de $30 \times 33 \mathrm{~mm}$ anteroposterior y laterolateral de la tráquea a nivel de $\mathrm{C} 7$ y $\mathrm{T} 1$ por debajo del cartílago cricoides, con un defecto en la pared posterior de la tráquea de $2.5 \mathrm{~mm}$, formando un trayecto fistuloso hacia la pared anterior del esófago, recuperando la interfase a nivel del segmento aórtico del esófago (Fig. 2).

Se programa para traqueostomía y reconstrucción esofágica. Se inicia el abordaje cervical transversal $2 \mathrm{~cm}$ por encima de la escotadura yugular y esternotomía media con sierra automática. Se identifican la fístula traqueoesofágica (Fig. 3) y las estructuras vasculares, troncos supraaórticos y sistemas venosos. Se identifica el defecto esofágico (Fig. 4) en su porción cervical, de aproximadamente $4 \mathrm{~cm}$ de diámetro. Se procede a realizar cierre primario del defecto con 


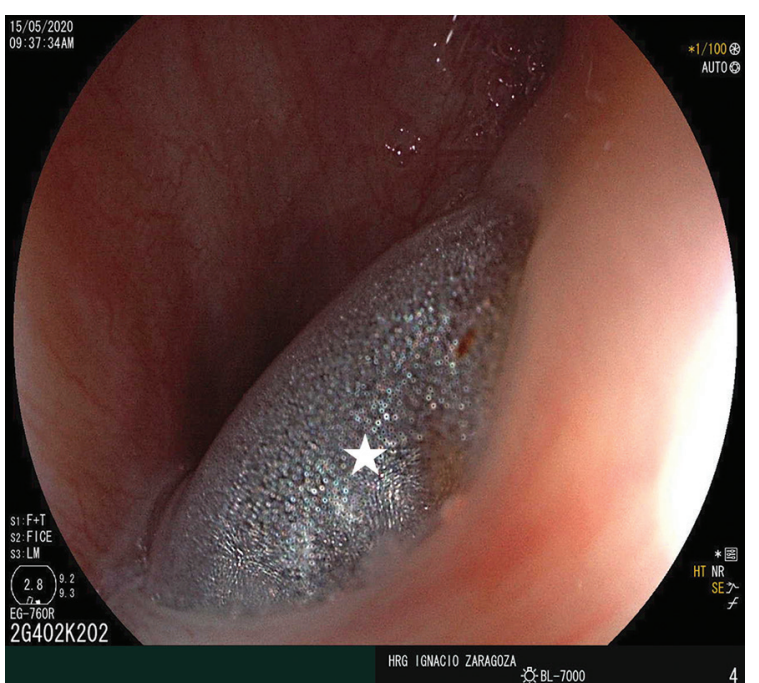

Figura 1. Imagen endoscópica en la que se observa la protrusión del balón (señalada con una estrella) del tubo endotraqueal hacia la luz esofágica.

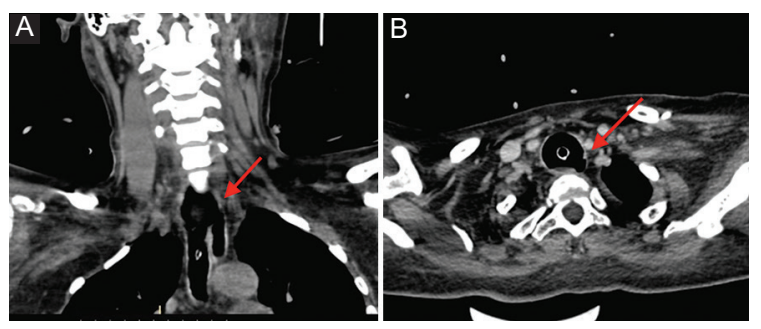

Figura 2. Tomografía computarizada en la que se observa la fístula entre la tráquea y el esófago a nivel de C7-T1 (flecha) como un trayecto hipodenso con densidad de gas. A: corte coronal. B: corte sagital.

sutura de Vicryl ${ }^{\circledR}$ 2-0 en un plano y posteriormente se coloca un parche muscular. Se procede a realizar traqueostomía en V, $5 \mathrm{~cm}$ por arriba de la carina, y se coloca una nueva cánula endotraqueal estéril (Fig. 5). Posteriormente se procede al desinflado del balón por parte del servicio de anestesiología, el retiro de la cánula orotraqueal con aspiración de secreciones y la reconexión al circuito de ventilación con la cánula endotraqueal previamente descrita. Se realizan resección traqueal y anastomosis terminoterminal con Prolene® 4-0 con puntos simples por fuera de la mucosa (Fig. 6). Se procede al cierre de la esternotomía con sutura de alambre con técnica hemi-Robicsek y al cierre de la cervicotomía por planos, previa colocación de drenaje. Lamentablemente, la paciente fallece en el posoperatorio inmediato debido a falla orgánica múltiple.

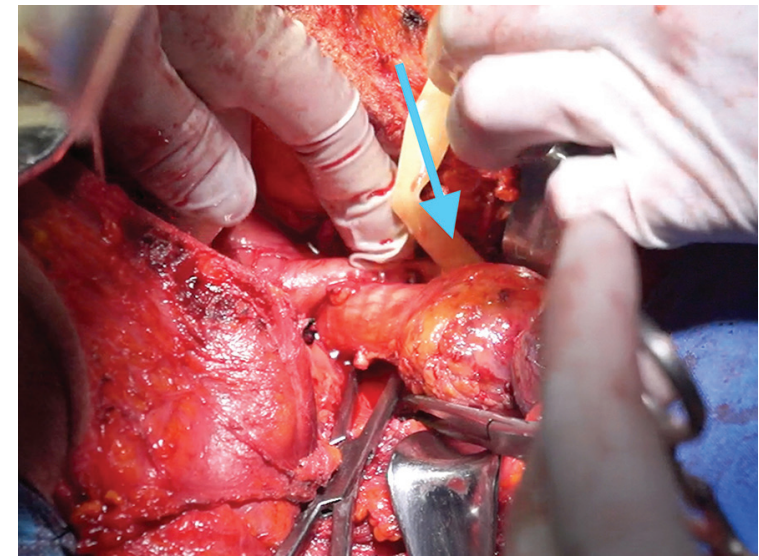

Figura 3. Imagen transoperatoria de la fístula traqueoesofágica secundaria a hiperinsuflación del balón endotraqueal (flecha), con una porción sana de tráquea distal por encima del tronco braquiocefálico.

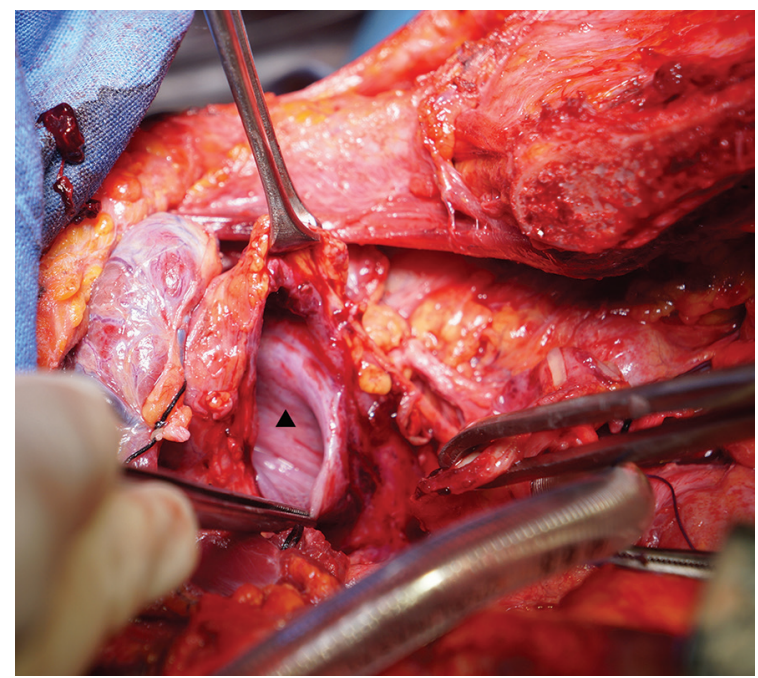

Figura 4. Imagen transoperatoria del defecto esofágico, por debajo del músculo constrictor de la faringe, con bordes revitalizados para permitir el cierre primario.

\section{Revisión de la literatura}

La tráquea es una estructura tubular cartilaginosa que conecta con la laringe en sentido rostral y con los bronquios principales en sentido caudal. El borde inferior del cartílago cricoides define el inicio de la tráquea, y la carina su término, normalmente a nivel del cuerpo vertebral T4. En promedio mide $11.8 \mathrm{~cm}$. Está formada por 18 a 22 anillos con cartílagos en forma de $\mathrm{C}$ y una pared membranosa posterior que conecta los extremos. Cada anillo traqueal tiene un promedio de $4 \mathrm{~mm}$ de altura y la pared tiene un espesor aproximado de $3 \mathrm{~mm}$. Los lóbulos derecho e 


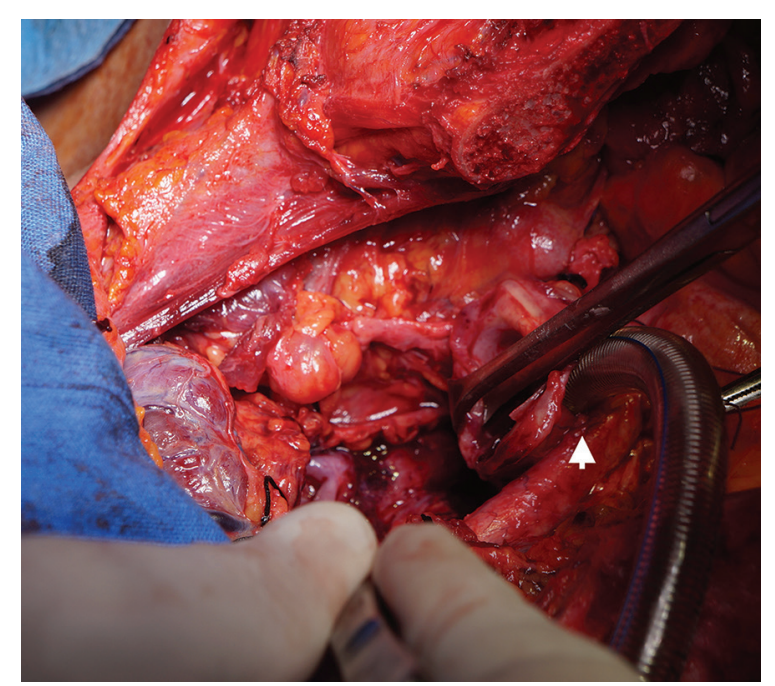

Figura 5. Imagen transoperatoria posterior a la resección de la porción traqueal fistulizada con traqueostomía en cabo distal (flecha) y cánula con alma de acero conectada al circuito de ventilación.

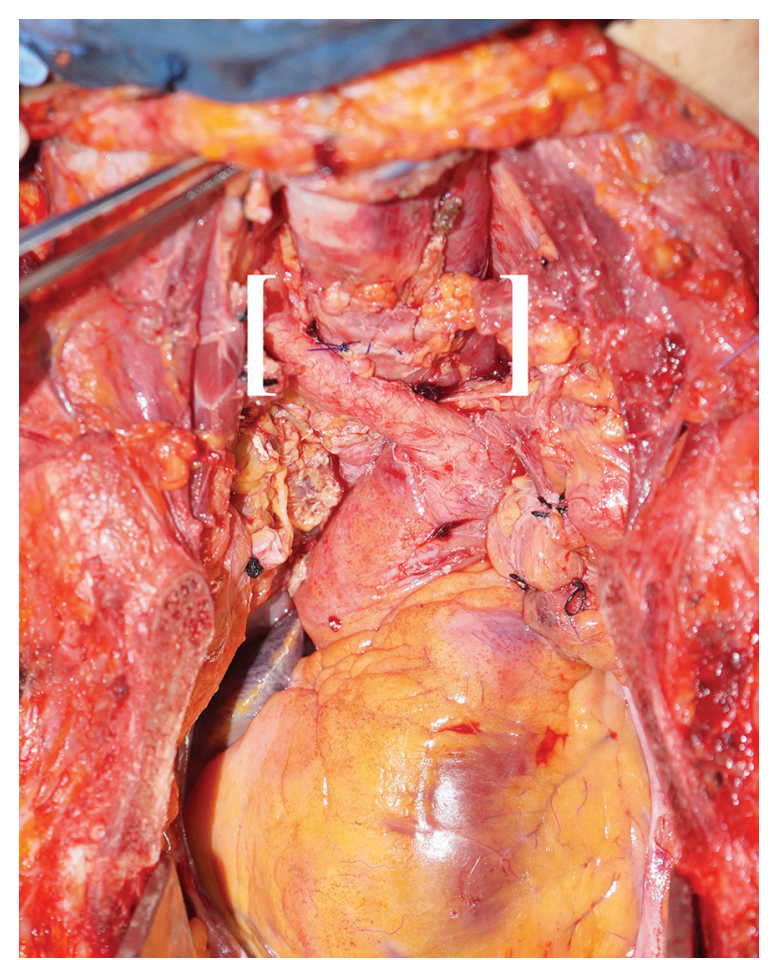

Figura 6. Imagen transoperatoria de la anastomosis término-terminal traqueal (entre corchetes), por encima del tronco braquiocefálico. La vena innominada izquierda fue seccionada.

izquierdo de la glándula tiroides se sitúan anterolaterales a la tráquea proximal y el istmo cruza anterior en el segundo o tercer anillo traqueal. El esófago se encuentra en íntima relación con la tráquea. El límite externo de la unión faringoesofágica es el cartílago cricoides. En la esofagoscopia, este se encuentra a $15 \mathrm{~cm}$ de la arcada dentaria. El esófago carece de serosa y consiste en una capa muscular longitudinal externa y una muscular circular interna. El esófago cervical tiene una longitud aproximada de 5-7 cm y contacta con la cara posterior del cartílago cricoides y la tráquea, anterior al músculo largo del cuello y los cuerpos vertebrales; desciende en contacto con la columna vertebral hasta la parte superior del mediastino posterior. Se relaciona por detrás con la columna cervical y los músculos prevertebrales, de los que se encuentra separado por un tejido celuloadiposo, lo que constituye un espacio de disección quirúrgica. Por delante está unido a la tráquea por unos tractos fibromusculares (músculo traqueoesofágico) fácilmente separables. El paquete neurovascular del cuello (arteria carótida común, vena yugular interna y nervio vago) discurre en una posición lateral y un poco anterior al esófago; el lóbulo lateral de la glándula tiroides se encuentra en contacto con el esófago, lo que requiere en ocasiones la ligadura y la sección de una o varias venas tiroideas. La lesión de los nervios laríngeos recurrentes, ya sea por sección completa o parcial, tracción, contusión, lesión térmica, isquemia o invasión tumoral, puede causar una paresia en las cuerdas vocales o parálisis que resulten en disfonía, pérdida completa de la voz o compromiso de la vía aérea, dependiendo de la extensión de la lesión y de la integridad del nervio contralateral. El cirujano debe ser cauteloso con el trayecto de estos nervios durante la disección de la tráquea proximal ${ }^{7-9}$.

La fístula traqueoesofágica debe sospecharse clínicamente si se presentan una distensión masiva del abdomen, secreciones anómalas o aspiración de contenido gastroalimentario desde el árbol traqueobronquial. La confirmación de la sospecha clínica puede realizarse mediante endoscopia superior, en la que se observa el balón endotraqueal protruyendo hacia la luz esofágica a través del trayecto fistuloso. La broncoscopia con fibra óptica debe efectuarse para definir el sitio exacto y las dimensiones de la fístula, así como para evidenciar estenosis laríngea o traqueal concomitante ${ }^{10,11}$.

Qureshi, et al. ${ }^{6}$ evaluaron la etiología y clasificaron según su anatomía a las fístulas aerodigestivas, proponiendo un algoritmo terapéutico divido en quirúrgico y no quirúrgico; éste último subdividido en conservador, endoprotésico y paliativo. La reparación debe ser anticipada con múltiples pasos esenciales preliminares, incluyendo el destete de la ventilación mecánica y el control de la comorbilidad asociada. Si 
no es posible el destete de la ventilación mecánica, la mayoría de los autores sugieren tomar medidas paliativas para prevenir complicaciones, tales como el retiro de la sonda nasogástrica, la colocación del balón de la cánula endotraqueal distal a la fístula, la gastrostomía derivativa y la enterostomía o la yeyunostomía para alimentación con el fin de disminuir el reflujo y la contaminación del árbol bronquial con contenido gástrico; esta última, además, permite favorecer el estado nutricional en conjunto con la administración de nutrición parenteral ${ }^{10,11}$. De esta manera se puede evitar ampliar la fístula y la contaminación de la vía aérea por el reflujo gastroesofágico, permitiendo continuar con la ventilación mecánica y una adecuada nutrición ${ }^{12}$.

La evaluación y la preparación prequirúrgica incluyen la realización de tomografía computarizada de laringe, tráquea y pulmones, broncoscopia flexible o rígida (o ambas) y endoscopia digestiva alta en todos los pacientes. Las infecciones pulmonares recurrentes y el control de la aspiración se tratan con fisioterapia, interrupción de la ingesta oral y antibióticos. La resolución espontánea es ilusoria, porque los bordes pueden estar epitelizados. La cirugía es el único tratamiento curativo y los objetivos principales que persigue son el cierre de la fístula y la prevención de su recurrencia ${ }^{3,5}$.

La elección de la técnica quirúrgica para la reparación de la fístula traqueoesofágica posintubación permanece aún en controversia. El abordaje quirúrgico puede ser mediante cervicotomía con o sin esternotomía media. La mayoría de las fístulas se encuentran la parte superior y media del esófago/tráquea, y se pueden operar a través de una incisión cervical baja. Si la fístula traqueoesofágica está ubicada distalmente, una esternotomía parcial puede mejorar la exposición de la carina. Las fístulas ubicadas en la carina, o muy distales en la tráquea, deben abordarse a través de una toracotomía derecha en el cuarto espacio intercostal. En consecuencia, las fístulas broncoesofágicas deben operarse mediante una toracotomía en el lado afectado ${ }^{5}$.

Una incisión baja de tipo «collar» y una esternotomía superior son suficientes para la mayoría de las reparaciones. La reparación se realiza mediante cierre directo con sutura en un solo acto quirúrgico, con resección traqueal y anastomosis, con cierre primario esofágico y sobreponiendo colgajos musculares. Si se prefiere, el abordaje puede ser en dos etapas, mediante derivación esofágica y cierre primario del defecto traqueal para posteriormente realizar un reemplazo esofágico mediante ascenso gástrico 0 transposición colónica. Otra opción es colocar stents esofágicos metálicos recubiertos autoexpandibles. La elección del método de reparación depende de las condiciones locales, la dependencia de la ventilación mecánica y las condiciones generales del paciente ${ }^{11}$.

Dhiwakar, et al. ${ }^{13}$ compararon distintas series de casos y describen algunos puntos clave en el manejo quirúrgico de la fístula traqueoesofágica: gastrostomía/yeyunostomía preoperatoria, estenosis traqueal concomitante, cicatrización de la fístula, mortalidad y seguimiento posoperatorio. Las complicaciones asociadas a la resección traqueal con anastomosis incluyen dehiscencia anastomótica, enfisema y neumomediastino ${ }^{14-16}$.

Las tasas de mortalidad van del $0.5 \%$ al $10.5 \%$; sin embargo, no son comparables de acuerdo con la etiología de la fístula, sea esta maligna o no. La mortalidad relacionada con complicaciones infecciosas debido a la recurrencia de la fístula o las pobres condiciones del paciente aumenta significativamente en relación con los pacientes sometidos a intervención quirúrgica aún dependientes de ventilación mecánica y con neumonía asociada ${ }^{11}$.

\section{Discusión}

Se describe en la literatura el tratamiento quirúrgico para la fístula traqueoesofágica como un procedimiento complejo, con elevada morbimortalidad. A pesar de que se recomienda el destete de la ventilación mecánica antes de la reparación, se han reportado algunos casos en los que se plantea el tratamiento definitivo a pesar de la situación crítica del paciente $e^{3,17}$.

Cuando el tratamiento quirúrgico definitivo no puede llevarse a cabo, se planean algunas estrategias que permitan la resolución de la sepsis pulmonar. Dichas estrategias incluyen la derivación esofágica y las endoprótesis traqueales en conjunto con un cierre temporal esofágico con parches musculares. El apoyo ventilatorio mecánico en el posoperatorio inmediato implica mala cicatrización, recurrencia de la fístula y riesgo de mediastinitis ${ }^{11,18}$.

Puma, et al. ${ }^{12}$ describieron dos pacientes que fueron sometidos a tratamiento quirúrgico, a pesar de los resultados insatisfactorios de la preparación preoperatoria prolongada, considerando lo siguiente: 1) aumento en el tamaño de la fístula y múltiples cuadros recurrentes de neumonía, requiriendo repetidamente 
el reinicio de la ventilación mecánica, previamente retirada; y 2) defecto distal que dificultaba la colocación del balón endotraqueal por debajo de la fístula, en un paciente con proceso de destete complicado debido a daño neurológico.

La cirugía debe ser considerada muy cautelosamente en los pacientes dependientes de ventilación mecánica, pero en opinión de los autores esta no debe ser considerada como una contraindicación absoluta para el cierre quirúrgico de la fístula traqueoesofágica. Cada caso debe evaluarse individualmente.

La mortalidad en estos pacientes alcanza un $18.9 \%$ a pesar de un adecuado tratamiento quirúrgico. Bayrak, et al. ${ }^{19}$ describen su experiencia con un paciente varón de 50 años que sufrió un accidente de tráfico y quedó cuadripléjico, con apoyo mecánico ventilatorio domiciliario, intervenido quirúrgicamente y egresado en el día 14 del posoperatorio, con sobrevida de 3 años posterior a la cirugía.

Por otro lado, Crema, et al..$^{20}$ describen un caso de un paciente varón de 27 años con ingesta de cáustico, que presentó 14 días después disfagia, odinofagia y dolor retroesternal. Se le realizó una endoscopia que evidenció una fístula amplia entre el esófago y el bronquio derecho, y se llevó a cabo tratamiento quirúrgico en dos tiempos, primero realizando esofagostomía cervical terminal y gastrostomía, con restitución del tránsito intestinal 8 meses después mediante faringocoloplastia.

La cirugía laringotraqueal está asociada con un mayor riesgo de complicaciones, relacionadas con la vía respiratoria, por lo que el manejo anestésico representa un desafío. Por ello, la comunicación multidisciplinaria abierta y continua optimiza los resultados. La planificación anestésica es relevante. Se ha documentado que los eventos adversos se llegan a presentar hasta en el $70 \%$ de los procedimientos quirúrgicos, incluyendo el transoperatorio y la recuperación ${ }^{21}$.

Cuando el anestesiólogo afronta un paciente con una fístula traqueoesofágica debe comenzar con una exhaustiva valoración preanestésica, con énfasis en la comorbilidad y en el tipo de la fístula. Debe prestar atención a la ventilación y la oxigenación en el preoperatorio y el posoperatorio, ya que se enfrenta a una vía aérea críticamente afectada. Se requiere una estrecha monitorización de los parámetros respiratorios y hemodinámicos para lograr una adecuada oxigenación y eliminación del dióxido de carbono, haciendo énfasis en el manejo farmacológico, con el fin de mejorar las condiciones quirúrgicas y mantener la estabilidad hemodinámica transoperatoria. Para ello debe canalizarse una vía arterial, preferentemente la arteria radial izquierda, pues la derecha recibe su perfusión del tronco braquiocefálico, que cruza la tráquea y puede ser comprimido durante la intervención ${ }^{22,23}$.

En pacientes extubados, no dependientes de soporte respiratorio, la tasa de éxito de la reparación quirúrgica es cercana al $90 \%$. La mortalidad operatoria es de aproximadamente un $13 \%$ y la tasa de recidiva es del $6.6 \%^{3}$. La abstención de tratamiento puede ser apropiada en situaciones sin expectativas de futuro, pero en una paciente joven, laboralmente activa y sin comorbilidad previa al evento cerebrovascular que condicionó su internamiento, se plantean infinidad de dudas, siendo cualquier opción de tratamiento susceptible de controversia.

Como conclusión, deben implementarse protocolos estructurados para la prevención de la fístula traqueoesofágica, incluyendo la selección adecuada del tubo endotraqueal, la revisión periódica de la presión de los balones endotraqueales (a ser posible cada 8 horas) con énfasis en mantener presiones menores de $25 \mathrm{mmHg}$, y considerar la traqueostomía temprana (en las primeras 2 semanas) ${ }^{24,25}$. En los casos en que, a pesar de estas medidas, se produzca una fístula traqueoesofágica, la preparación adecuada, la selección del abordaje quirúrgico y la vigilancia del posoperatorio mejoran el pronóstico de los pacientes.

\section{Agradecimientos}

Los autores agradecen a los servicios de anestesiología, imagenología y medicina interna, por su siempre incondicional apoyo.

\section{Financiamiento}

Los autores no recibieron patrocinio para llevar a cabo este artículo.

\section{Conflicto de intereses}

Los autores declaran no tener conflicto de intereses alguno.

\section{Responsabilidades éticas}

Protección de personas y animales. Los autores declaran que para esta investigación no se han realizado experimentos en seres humanos ni en animales. 
Confidencialidad de los datos. Los autores declaran que han seguido los protocolos de su centro de trabajo sobre la publicación de datos de pacientes.

Derecho a la privacidad y consentimiento informado. Los autores han obtenido el consentimiento informado de los pacientes y/o sujetos referidos en el artículo. Este documento obra en poder del autor de correspondencia.

\section{Bibliografía}

1. Kaur D, Anand S, Sharma P, Kumar A. Early presentation of postintubation tracheoesophageal fistula: perioperative anesthetic management. J Anaesthesiol Clin Pharmacol. 2012;28:114-6.

2. Bolca C, Păvăloiu V, Fotache G, Dumitrescu M, Bobocea A, Alexe M, et al. Postintubation tracheoesophageal fistula - diagnosis, treatment and prognosis. Chirurgia (Bucur). 2017;112:696-704.

3. De Castro G, Iribarren M, Rivo E, Meléndez R, Nóvoa E, Cañizares M, et al. Fístula traqueoesofágica en paciente intubado: tratamiento mediante exclusión y patch esofágico. Cir Esp. 2005;77:230-2.

4. Paraschiv M. Tracheoesophageal fistula - a complication of prolonged tracheal intubation. J Med Life. 2014;7:516-21.

5. Bibas BJ, Cardoso PF, Minamoto H, Pêgo-Fernandes PM. Surgery for intrathoracic tracheoesophageal and bronchoesophageal fistula. Ann Transl Med. 2018;6:210

6. Qureshi YA, Muntzer Mughal M, Fragkos KC, Lawrence D, George J, Mohammadi B, et al. Acquired adult aerodigestive fistula: classification and management. J Gastrointest Surg. 2018;22:1785-94.

7. Furlow PW, Mathisen DJ. Surgical anatomy of the trachea. Ann Cardiothorac Surg. 2018;7:255-60.

8. Benjamin ER, Inaba K. Cervical esophagus. En: Demetriades D, Inaba $\mathrm{K}$, Velmahos $\mathrm{G}$, editores. Atlas of surgical techniques in trauma. Cambridge: Cambridge University Press; 2015. p. 101-6.

9. Durand-Fontanier S, Valleix D. Anatomie chirurgicale de l'œsophage. EMC (Elsevier Masson SAS, Paris), Techniques chirurgicales - Appareil digestif, 40-170; 2007.
10. Athanassiadi K, Gerazounis M. Repair of postintubation tracheoesophageal fistula in polytrauma patients. Injury. 2005;36:897-9.

11. Foroulis CN, Nana C, Kleontas A, Tagarakis G, Karapanagiotidis GT, Zarogoulidis $\mathrm{P}$, et al. Repair of post-intubation tracheoesophageal fistulae through the left pre-sternocleidomastoid approach: a recent case series of 13 patients. J Thorac Dis. 2015;7(Suppl 1):S20-6.

12. Puma F, Vannucci J, Santoprete S, Urbani M, Cagini L, Andolfi M, et al. Surgery and perioperative management for post-intubation tracheoesophageal fistula: case series analysis. J Thorac Dis. 2017;9:278-86.

13. Dhiwakar M, Ronen O, Supriya M, Mehta S. Surgical repair of mechanical ventilation induced tracheoesophageal fistula. Eur Arch Otorhinolaryngol. 2020;277:323-31.

14. Vieth M, Martínez F. Tratamiento quirúrgico de la fístula traqueoesofágica adquirida. Reporte de caso. Rev Virtual Posgrado. 2016;1:1-9.

15. Muniappan A, Mathisen DJ. Repair of tracheo-oesophageal fistula. Multimed Man Cardiothorac Surg. 2016;2016:mmw002.

16. Lee JE, Chang MY, Kim KH, Jung YH. Post-intubation tracheoesophageal fistula with posterior glottic web. Clin Exp Otorhinolaryngol. 2011;4:105-8.

17. Collier KP, Zubarik RS, Lewis JH. Tracheoesophageal fistula from an indwelling endotracheal tube balloon: a report of two cases and review. Gastrointest Endosc. 2000;51:231-4.

18. Mathisen DJ, Grillo HC, Wain JC, Hilgenberg AD. Management of acquired nonmalignant tracheoesophageal fistula. Ann Thorac Surg. 1991:52:759-65.

19. Bayrak Y, Tanju S, Suoglu Y, Toker A. Treatment of a benign tracheoesophageal fistula in a ventilator dependent quadriplegic patient. Thorac Cardiovasc Surg. 2006;54:554-5.

20. Crema E, Fatureto MC, Gonzaga MN, Pastore R, Da Silva AA. Tracheoesophageal fistula after caustic ingestion. J Bras Pneumol. 2007;33:105-8.

21. Pearson KL, McGuire BE. Anaesthesia for laryngo-tracheal surgery, including tubeless field techniques. BJA Education. 2017;17:242-8.

22. Benumoff JL. Anesthesia for special elective therapeutic procedures. En: Benumoff JL, editor. Anesthesia for thoracic surgery. $2^{\text {nd }}$ ed. Philadelphia: W.B. Saunders; 1995. p. 530-41.

23. Dogar SA, Hamid M. Intraoperative ventilatory management of adult tracheoesophageal fistula. J Pak Med Assoc. 2016;66:1176-8.

24. Calvo Medina V, Galán Gil G, Sales Badía G, Morcillo Aixelá A, García Zarza A, Tarrazona Hervás V, et al. Fístulas traqueoesofágicas benignas adquiridas. Arch Bronconeumol. 1997;33:577-81.

25. Mooty RC, Rath P, Self M, Dunn E, Mangram A. Review of tracheo-esophageal fistula associated with endotracheal intubation. J Surg Educ. 2007;64:237-40. 